\title{
THE INTERPRETATION OF PHOTOELECTRIC COLORS FOR STARS OF TYPES B-F
}

\author{
W. K. Bonsack, J. L. Greenstein, J. S. Mathis, W. G. Melbourne, G. Neuge- \\ bauer, R. L. Newburn, K. H. Olsen, W. G. Tifft, H. D. Wahlquist, \\ AND G. WALLERSTEIN \\ Mount Wilson and Palomar Observatories \\ Carnegie Institution of Washington, California Institute of Technology \\ Received September 14, 1956
}

\section{ABSTRACT}

The accumulation of photoelectric data on the Johnson-Morgan system of $B-V$ and $U-B$ colors makes a preliminary theoretical reconnaissance desirable The colors were predicted for atmospheres of a wide range of effective temperatures and electron pressures. The effects of the Balmer jump on the response in the $U$ band and of the Balmer lines in the $B$ band were included, using averages taken over spectral type and luminosity classes. Table 2 gives the predicted fluxes as compared to a black body, the corrected $B-V$ and $U-B$ colors, and the color temperatures The zero point is based on Code's spectral scans of two stars. The results are most useful for differential effects over small ranges of $\theta$ and $P_{e}$; the general temperature and pressure scale derived colorimetrically seems reasonable The large effect of lines in certain white dwarfs explains some features of the observed colors.

\section{INTRODUCTION}

The details of the continuous spectra of the sun and stars have provided information about and are correlated with the temperature, $T$, surface gravity, $g$, and composition of stellar atmospheres. Interpretation of the observed flux, color, gradient, and Balmer discontinuity has been quite successful. The growth of precise multicolor photoelectric photometry has permitted distinctions to be made between space reddening and temperature reddening, between stars of the two population types, and even permitted colorimetric identification of the white dwarfs. A complete explanation of the observed colors requires detailed atmospheric models, not available, for a wide range of $T, g$, and composition. The rapid growth of data available on the Johnson $U, B, V$ system suggested the value of this preliminary reconnaissance, which was made by a group of graduate students at the California Institute of Technology.

The Johnson system provides two color indices: $B-V$, which is largely determined by the temperature, and $U-B$, which is affected by the Balmer discontinuity. For normal stars there is only small scatter from the mean curve in the $U-B, B-V$ plane; these colors are well correlated with spectral type; white dwarfs and subdwarfs (and weak-line stars slightly) deviate from the mean curve. This curve clearly shows the effect of the Balmer discontinuity in depressing the $U$ point; the white dwarfs lie more nearly on a straight line such as might be expected from a black body. The small scatter indicates that normal stars have only a small spread in composition, $T$, and $g$ as a function of spectral type. A given point in the $U-B, B-V$ plane is determined uniquely by these parameters.

A three-color system cannot rival the information available for the sun or for stars from the combination of spectral scanning, such as is now being carried out by Whitford and Code, and spectrographic analysis. Consequently, for a first reconnaissance we adopted a set of simplifying assumptions for the theoretical stellar atmospheres. The composition was held constant, the temperature distribution in an unperturbed gray atmosphere in radiative equilibrium was adopted, and then $\kappa / \kappa_{\nu}$ was assumed independent of depth; the models were characterized by $T_{e}$ and $P_{e}$ (effective temperature and effective mean electron pressure) rather than by $T_{e}$ and $g$. Transformation to the latter 
is possible on the further assumption of hydrostatic equilibrium and an effective level of origin of the continuum. We find that line absorption has very large effects, because of the location and breadth of the photoelectric response-curves. We have computed the integrated response in the $U, B, V$ bands for each $T_{e}$ and $P_{e}$ and applied estimated corrections for the hydrogen lines. No attempt was made to include the effects of metallic lines. These integrations give magnitudes with an arbitrary zero point which must be evaluated either from stars of known flux or from external knowledge of the correlation between spectral type, $T_{e}, P_{e}$, and colors.

\section{COMPUTATIONS AND RESULTS}

\section{a) Total Flux and Magnitudes}

Let $F(\lambda)$ denote the flux received at the top of the atmosphere from a star. Let $\phi(\lambda)$ denote the response function of a phototube plus filter plus telescope system to be used to measure the flux. Then the total flux, $R$, recorded by the system will be given by

$$
R=\int_{0}^{\infty} F(\lambda) \phi(\lambda) d \lambda .
$$

We neglect atmospheric effects, since we wish to deal theoretically with colors reduced to outside the atmosphere, in accordance with nearly all present-day photoelectric photometry. We convert total flux values to a magnitude scale by taking

$$
m(R)=-2.5 \log _{10} R .
$$

If we compute $R$ for different response functions corresponding to different colors, we can define color indices by taking differences between the response magnitudes in different colors. The zero point of the color system so established is arbitrary and must be fixed by separate considerations.

Since the accuracy of the models employed to determine $F(\lambda)$ scarcely warrants high computational accuracy, the integrals in equation (1) were performed in an approximate manner. We expanded $F(\lambda)$ as a Taylor series about a wave length $\lambda_{0}$, defined by

$$
\lambda_{0}=\frac{\int_{0}^{\infty} \lambda \phi(\lambda) d \lambda}{\int_{0}^{\infty} \phi(\lambda) d \lambda}
$$

which is so chosen that the contribution to $R$ from the first-derivative term in the expansion vanishes; i.e., the integral becomes

$R=\int_{0}^{\infty}\left[F\left(\lambda_{0}\right)+\left.\frac{d F(\lambda)}{d \lambda}\right|_{\lambda_{0}}\left(\lambda-\lambda_{0}\right)+\left.\frac{1}{2} \frac{d^{2} F(\lambda)}{d \lambda^{2}}\right|_{\lambda_{0}}\left(\lambda-\lambda_{0}\right)^{2}+\ldots\right] \phi(\lambda) d \lambda$.

Integrating term by term and using the definition of $\lambda_{0}$, the first-derivative term cancels, to yield

$$
\dot{R}=F\left(\lambda_{0}\right) \int_{0}^{\infty} \phi(\lambda) d \lambda+\left.\frac{1}{2} \frac{d^{2} F(\lambda)}{d \lambda^{2}}\right|_{\lambda_{0}}\left[\int_{0}^{\infty} \lambda^{2} \phi(\lambda) d \lambda-\lambda_{0}^{2}\right]+\ldots .
$$

Second- and higher-derivative terms become small not only because of the factorial coefficients but because terms like that in the parentheses in equation (5), which is the difference between the second moment of $\lambda$ and the square of the first moment, tend to zero. To the first order, then,

$$
R=F\left(\lambda_{0}\right) \int_{0}^{\infty} \phi(\lambda) d \lambda .
$$


As a check on this approximation, two computations were carried out by direct integration of $F\left(\theta, P_{e}\right)$ according to equation (1) and compared with the approximate values according to equation (6). The greatest deviation observed was 5 per cent in $R$. These computations were carried out for values of $\theta$ and $P_{e}$ where the approximation seemed least likely to be valid.

Direct numerical integration according to the definition in equation (3) yields the values of $\lambda_{0}$. The response function used was that given by Johnson and Morgan (1951), with corrections to include two aluminum reflections. The ultraviolet band was considered in two parts, divided at the Balmer limit. This step was necessary because of the obvious failure, at a discontinuity in $F(\lambda)$, of the approximate form used to replace the integral. Thus, for the ultraviolet, equation (6) becomes a sum of two terms,

$$
R(U)=F\left(U_{1}\right) \int_{0}^{3650} \phi_{U}(\lambda) d \lambda+F\left(U_{2}\right) \int_{3650}^{\infty} \phi_{U}(\lambda) d \lambda,
$$

where $F\left(U_{1}\right)$ and $F\left(U_{2}\right)$ are, respectively, the fluxes at the effective wave lengths in the short- and long-wave-length parts of the ultraviolet band; and $\phi_{U}(\lambda)$ is $\phi(\lambda)$ in the ultraviolet band.

We write equation (7) henceforth as

$$
R(U)=F\left(U_{1}\right) w_{1}+F\left(U_{2}\right) w_{2} .
$$

The numbers $w_{1}$ and $w_{2}$ can be interpreted as relative weights assigned to the two portions of the ultraviolet response band. The effect of the higher Balmer lines was approximately accounted for by modifying these weights slightly, as discussed in part $d$ of this section.

The vaues of $\lambda_{0}$ obtaned were $\lambda_{U}^{(1)}=3388 \mathrm{~A}, \lambda_{U}^{(2)}=3773 \mathrm{~A}, \lambda_{B}=4430 \mathrm{~A}$, and $\lambda_{V}=$ 5540 A.

\section{b) The Basic Method}

The $F$ which appears in equations (6) and (8) must be determined at the effective wave length, $\lambda_{0}$, as a function of the temperature and the electron pressure, $P_{e}$. The procedure involved using Vitense's tables (1951), which give the opacity $\kappa_{\nu}$ and the Rosseland mean $\bar{\kappa}$ as functions of $\theta\left(=5040^{\circ} / T\right)$, electron pressure, and wave length. Assuming a variety of physical conditions, Vitense computed the opacity, from all known sources, of a mixture of elements representative of stellar material. Since for the ranges of temperature and electron pressure considered in this paper the opacity due to hydrogen or the negative hydrogen ion is always predominant, the details of the heavy-element composition of the mixture are unimportant. For low values of $\theta$, Vitense's values of $\kappa_{\nu}$ and $\bar{\kappa}$ were checked by using the newly computed atomic opacities given by Ueno (1954) and by Ueno, Saito, and Jugaku (1954).

With values of $\bar{\kappa} / \kappa_{\nu}$ and $h c / \lambda_{0} k T$ we then determined the ratios $F(\lambda) / B(\lambda)$ from tables given by Burkhardt (1939) and by Chandrasekhar (1950). The Burkhardt tables are based on Eddington's solution of the Schwarzschild integral equation for the source function in a gray atmosphere. They were entered by using both analytical interpolation and Münch's (1945) graphical representation. It would have been interesting to compare the results of our computation with those that might be obtained with the Chandrasekhar (flux-weighted) or Planck mean opacities. Unfortunately, no detailed tables of the required $\bar{\kappa}$ for $\mathrm{H}, \mathrm{H}^{-}, \mathrm{He}$ I, He II, and other sources have yet been published.

\section{c) Color Indices}

Following definition (2), we can represent a color index by

$$
C(b, a)=-2.5[\log R(b)-\log R(a)]+c_{1} .
$$


Here $c_{1}$ represents the zero-point constant to convert to some standard color system. Substituting the proper values of $R$, we directly deduce formulae representing $(B-V)$ and $(U-B)$ as follows:

$$
\begin{aligned}
& B-V=-25\left[\log \frac{F(B)}{B(B)}-\log \frac{F(V)}{B(V)}+\log \frac{B(B)}{B(V)}\right]+c_{2}, \\
& \begin{aligned}
U-B=-2.5\left\{\log \left[w_{1} \frac{F\left(U_{1}\right)}{B\left(U_{1}\right)}+w_{2} \frac{B\left(U_{2}\right)}{B\left(U_{1}\right) B} \frac{F\left(U_{2}\right)}{\left(U_{2}\right)}\right]\right. \\
\left.\quad-\log \frac{F(B)}{B(B)}+\log \frac{B\left(U_{1}\right)}{B(B)}\right\}+c_{3},
\end{aligned}
\end{aligned}
$$

where the constants contributed by the response integrals are combined with $c_{1}$ to give new zero-point constants, $c_{2}$ and $c_{3}$. For convenience in computation, all fluxes are expressed as ratios to the Planck function, $B$, at the effective temperature and the wave length, $\lambda_{0}$, of the relevant filter band.

\section{d) Line Corrections}

In deriving values of $F(\lambda) / B(\lambda)$ from values of $\bar{\kappa} / \kappa_{\nu}$ and proceeding from these to colors according to the equations (10) and (11), one cannot expect to reproduce observational results because the absorption lines, which significantly affect the colors of all but the hottest stars, have been ignored. Instead of correcting exactly for all lines in the spectrum, we have restricted ourselves to the hydrogen lines, the strongest lines in the spectra of all but the coolest stars treated. We hoped to introduce a correction of at least reasonable magnitude and varying properly with spectral and luminosity class.

Data on the equivalent widths of the hydrogen lines for 95 stars of spectral classes $\mathrm{O}$ to F8 are given by Günther (1933). These have been shown to be nearly correct by Stock (1956). These data were chosen because of the large number of stars of spectral types of interest in this study and the large number of Balmer lines measured in each star. While the measures of Günther do not approach in accuracy modern measures of equivalent widths made with higher-dispersion equipment, no modern study exists of such a large number of stars on an internally consistent scheme.

The correction for line absorption was made by different methods in the blue and ultraviolet bands. No correction was made in the yellow band, since $\mathrm{H} \alpha$ and $\mathrm{H} \beta$ are in the far wings of the sensitivity function, where their effect is negligible. The blue band includes in principle all the Balmer lines except $\mathrm{Ha}$, but the contribution of lines to the shortward of about $3800 \mathrm{~A}$ is negligible. The ultraviolet band overlaps the blue band and has equal response at about $\mathrm{H} \epsilon$. It was therefore decided to correct the blue band for the individual resolvable Balmer lines and the ultraviolet band for the unresolved lines near the limit.

The blue band.-Günther gives equivalent widths for Balmer lines to H14 for some stars, complete to $\mathrm{H} 9$ for all stars, except for seven of the later-type stars in his list. It was therefore decided to consider all lines from $\mathrm{H} \beta$ to $\mathrm{H} 9$ as resolvable and to correct the flux passed by the blue filter for them. Average equivalent widths for the Balmer lines were derived from Günther's data for stars of the spectral and luminosity classes represented by the values of temperature and electron pressure used in this paper.

The first problem, then, was to correlate the values of temperature and electron pressure with spectral and luminosity classes. A correlation of effective temperature with spectral class is given by Keenan and Morgan (1951); this was used directly. In assigning temperature and electron pressure to a luminosity class, we were guided by the results of Chandrasekhar and Münch (1946), who computed the Balmer discontinuity for various electron pressures as a function of spectral class and compared the results with the values observed by Barbier and Chalonge for main-sequence stars. Electron pressures 
lower than those indicated by this study for main-sequence stars were taken to correspond to giant and supergiant stars. For most stars of interest, classifications were found in Johnson and Morgan (1953) and in Keenan and Morgan (1951).

To get mean equivalent widths for the spectral and luminosity classes of interest for Günther's data, we found it necessary to average stars of the proper luminosity class within one spectral subdivision of the desired spectral class and smooth the resulting curve. Smoothed equivalent widths were converted to fluxes by multiplying by the energy in the continuum-taken to be that of a black body at the temperature corresponding to the appropriate spectral class-and by the instrument function, $\phi(\lambda)$, at that wave length of the line. The line fluxes were summed over all lines considered, and the sum was subtracted from the total flux, $R$, in the blue band. For some values of temperature and pressure the total line flux could not be derived from Günther's data, because such combinations of $\theta$ and $P_{e}$ do not correspond to real stars. In these cases the line corrections were estimated by extrapolation to the desired $\theta$, at a given $P_{e}$. Such corrections are inclosed in parentheses in Table 2.

The ultraviolet band.- The method of correction in the ultraviolet has its basis in the method by which the integral response in equation (1) was approximated. As previously stated, the ultraviolet band was divided at the Balmer limit, and the fluxes at the effective wave length of each part were recombined according to equation (8), where the weights $w_{1}$ and $w_{2}$ were defined as follows:

$$
w_{1}=\int_{0}^{3650} \phi_{U}(\lambda) d \lambda, \quad w_{2}=\int_{3650}^{\infty} \phi_{U}(\lambda) d \lambda .
$$

In actual practice these weights were normalized to sum to unity. This changes only the zero-point constant, which is arbitrary in any case.

Barbier and Chalonge (1939) introduced the use of the parameter $\lambda_{1}$ to describe the energy distribution in the ultraviolet continuum of stellar spectra; it is defined as the wave length at which the apparent continuum has dropped to halfway between the heights of the true continuum shortward and longward of the Balmer limit. This "effective Balmer limit" is longward of the true limit because the higher Balmer lines overlap significantly and the apparent continuum drops from the high to the low value as the overlapping increases. Stronger Balmer lines give a larger value of $\lambda_{1}$. From the definitions of $w_{1}$ and $w_{2}$ it is seen that, if $\lambda_{1}$ or something similar were used instead of the Balmer limit as the limit of integration, the low flux value, $F\left(U_{1}\right)$, shortward of the Balmer limit would have greater effect, as it should, on the total ultraviolet flux; its relative importance would depend on the strengths of the Balmer lines. Barbier and Divan (1952) have given an extensive list of values of $\lambda_{1}$ which might be considered appropriate substitutes for the Balmer limit used in the weight equation above. These values, however, do not adequately distinguish between giants and dwarfs.

A basically similar way to choose a wave length at which to divide the ultraviolet band was finally chosen. If one approximates the profiles of the Balmer lines by triangles, then at that point in the series where the equivalent width is equal to half the separation of the line centers, the apparent continuum should be near the true value longward of the limit; at that point where the equivalent width is twice the line separation, the apparent continuum should be reduced to its value shortward of the Balmer limit. The wave length halfway between these points was chosen as the effective Balmer limit. It should be noted here that the effective wave length of each part of the ultraviolet band was not varied, but the ones determined by dividing at the Balmer limit were retained throughout.

Unfortunately, overlapping in higher Balmer lines makes it impossible to obtain measured equivalent widths to use in the foregoing procedure. It was therefore assumed on the basis of the theory of the Stark effect that the equivalent widths were essentially 
constant among the later members of the series, and the width of $\mathrm{H} \delta$ was chosen as typical of all later series members. The mean equivalent width of $\mathrm{H} \delta$ for each spectral and luminosity class considered was used for this purpose. From this, by the method of analysis outlined here, a "theoretical" value of $\lambda_{1}$, the effective Balmer limit, was derived for each spectral and luminosity class, and a pair of weights, $w_{1}$ and $w_{2}$, was derived for each such $\lambda_{1}$. These weights were associated with the proper temperature and electron pressure as stated in the section on the blue band, and a value of the total flux, $R$, in the ultraviolet band, corrected for lines, was obtained for each combination of temperature and electron pressure.

It is recognized that the corrections for lines in both bands are highly approximateespecially so in the ultraviolet. Fluxes in lines should not be simply subtracted, but rather the line-absorption coefficients should be added and the blended lines synthesized. Our predicted colors may still be considerably different from the observed values, but at least they should be closer than if no line correction had been applied.

\section{e) Computing Formulae and Results}

When the line corrections are included according to the previous discussion, formulae (10) and (11) become

$$
\begin{aligned}
& B-V=-2.5\left\{\log \left[\frac{F(B)}{B(B)}-\sum_{H} \frac{W(H) \phi(H) B(H)}{B(B) \int_{0}^{\infty} \phi_{B}(\lambda) d \lambda}\right]\right. \\
& \left.-\log \frac{F(V)}{B(V)}+\log \frac{B(B)}{B(V)}\right\}+c_{2}^{\prime} \\
& U-B=-2.5\left\{\log \left[w_{1} \frac{F\left(U_{1}\right)}{B\left(U_{1}\right)}+w_{2} \frac{B\left(U_{2}\right) F\left(U_{2}\right)}{B\left(U_{1}\right) B\left(U_{2}\right)}\right]\right. \\
& \left.-\log \left[\frac{F(B)}{B(\bar{B})}-\sum_{H} \frac{W(H) \phi(H) B(H)}{B(B) \int_{0}^{\infty} \phi_{B}(\lambda) d \lambda}\right]+\log \frac{B\left(U_{1}\right)}{B(B)}\right\}+c_{3}^{\prime},
\end{aligned}
$$

where $W(H)$ is the equivalent width of a particular line considered and $\phi(H)$ and $B(H)$ are $\phi(\lambda)$ and $B(\lambda)$ at the line. Formulae (10), (11), (13), and (14) are the ones upon which our color computations have been based. In Table 2 the summations over the lines in equations (13) and (14) are abbreviated as $\Sigma_{H}$.

To determine the zero-point constants, $c_{2}^{\prime}$ and $c_{3}^{\prime}$, we have made use of relative flux scans, photoelectrically observed by Code (1955). In particular, we have used the stars $\sigma$ Boo (F2 V) and $a$ Vir (B1 V). The latter was chosen because of relative freedom from line absorption. For $\sigma$ Boo, Code has given the flux corrected for line absorption as well as the directly observed values. The values of the relative flux, in logarithms, at the effective wave lengths used in this paper are given in Table 1 . When the observed relative flux values are used in equations (13) and (14) and the Planck function eliminated, we can derive $(B-V)-c_{2}^{\prime}$ and $(U-B)-c_{3}$ for the stars considered. The line corrections are, of course, directly integrated into the observations. The weights for the ultraviolet band were determined by noting the wave length of the middle of the Balmer jump from Code's observations and using equation (12). The derived values of $(B-V)-c_{2}^{\prime}$ and $(U-B)-c_{3}^{\prime}$ were compared directly with the average colors for B1 V and $\mathrm{F} 2 \mathrm{~V}$ stars as given by Johnson and Morgan (1953) and the average values of $c_{2}^{\prime}$ and $c_{3}^{\prime}$ derived. The deviations of the predicted colors, given in Table 1 , from the mean values given by 
Johnson and Morgan were found to be 0.04 mag. in $(B-V)$ and 0.13 mag. in $(U-B)$. The adopted constants were $c_{2}^{\prime}=+0.520$ and $c_{3}^{\prime}=-0.335$.

The relative flux values for $\sigma$ Boo corrected for lines provide a measure of the effect of lines at spectral class F2. When the corrected flux values are used in equations (13) and (14), the resultant point is found to move strongly toward negative values in both color indices, as shown by the vector in Figure 1 and in Table 1 , of $(B-V)$ versus $(U-B)$. The model-atmosphere values of $\theta_{e}=0.74$ and $\log P_{e}=+1.89$ for $\sigma$ Boo as given by Code are in good agreement with the position of the star among the curves computed in this paper. It will be noted that the observed position of the stars $a$ Vir and $\sigma$ Boo is such, with this zero point, that they are above and below the observed mean curve in the $U-B$ co-ordinate and at about the correct spectral type in the $B-V$ co-ordinate. This seems the best single compromise, if a fixed zero point is to be used for the range from B- to F-type stars.

In Table 2, which includes the results of the computations, sufficient detail has been given that suitable modifications of the correction for lines, $\Sigma_{H}$, and for weights in the ultraviolet bands can easily be made. In addition, it should be noted that in Table 2 and in the figures the particular zero-point constants $c_{2}^{\prime}=+0.520$ and $c_{3}^{\prime}=-0.335$ were

TABLE 1

Relative Flux BASEd on Spectral Scans By Code (1955)

\begin{tabular}{|c|c|c|c|c|c|c|c|c|}
\hline \multirow{2}{*}{ StAR } & \multicolumn{4}{|c|}{ LOG $\mathrm{F}\left(\lambda_{0}\right) / \mathrm{F}_{0} *$} & \multirow{2}{*}{$w_{1}$} & \multirow{2}{*}{$w_{2}$} & \multirow{2}{*}{$\begin{array}{l}(B-V) \\
(\mathrm{Mag})\end{array}$} & \multirow{2}{*}{$\begin{array}{l}(U-B) \\
(\text { Mag.) }\end{array}$} \\
\hline & $U_{1}$ & $U_{2}$ & $B$ & V & & & & \\
\hline $\begin{array}{l}\sigma \text { Boo (obs) } \\
\sigma \text { Boo (corr) } \\
\alpha \text { Vir (obs) }\end{array}$ & $\begin{array}{l}-0280 \\
-\quad 136 \\
+0656\end{array}$ & $\begin{array}{l}-0176 \\
+\quad 056 \\
+0500\end{array}$ & $\begin{array}{l}-0068 \\
+\quad 016 \\
+0304\end{array}$ & $\begin{array}{r}-0144 \\
-\quad 132 \\
0000\end{array}$ & $\begin{array}{l}0773 \\
\quad 773 \\
0 \quad 756\end{array}$ & $\begin{array}{ll}0 & 227 \\
& 227 \\
0 & 244\end{array}$ & $\begin{array}{l}+033 \\
+\quad 15 \\
-024\end{array}$ & $\begin{array}{ll}+0 & 13 \\
-0 & 08 \\
-1 & 13\end{array}$ \\
\hline
\end{tabular}

$* F_{0}$ is the arbitrary zero point used by Code in expressing the relative energy distribution for a given star

used. Thus the zero point can be changed by additive constants to all $B-V$ and $U-B$ values. However, since the strength of the metallic lines and their effect on the line corrections begin to increase rapidly for $\theta_{e}>0.56$, the true zero-point corrections can properly be represented only by a vector field. A zero point based, for example, on the theoretical solar flux would be incorrect for F stars; the zero points may be seriously affected by differences in composition, as shown for the subdwarfs (Schwarzschild, Searle, and Howard 1955). Because of the uncertainty of the zero point, the greatest value of our computations would be in study of differential effects over a small range of spectral type, e.g., RR Lyr stars, and the study of the effects of surface gravity or composition.

Figure 1 shows the results in summary form. The mean observed $B-V$ and $U-B$ relation for main-sequence stars is plotted, with mean spectral types. Theoretical curves are given for $\log P_{e}=+2,+3$, and +4 , and each plotted point is labeled by a value of $\theta_{e}$. In addition, the observed and line-free positions given by data of spectral scans for $a$ Vir and $\sigma$ Boo are shown. Figure 2 shows an enlarged section around B5-F0 to display the complicated run of the loci of stars of constant temperature and the sensitive dependence of the colors on pressure.

Figure 3 contains the observed curve and the locus (labeled by values of $\theta$ ) for the predicted colors of black bodies. The last two columns of Table 2 contain the $\theta$ for blackbody radiation corresponding to the predicted $U-B$ and $B-V$ colors and, together with the previous data, permit construction of the color-effective-temperature relations. 


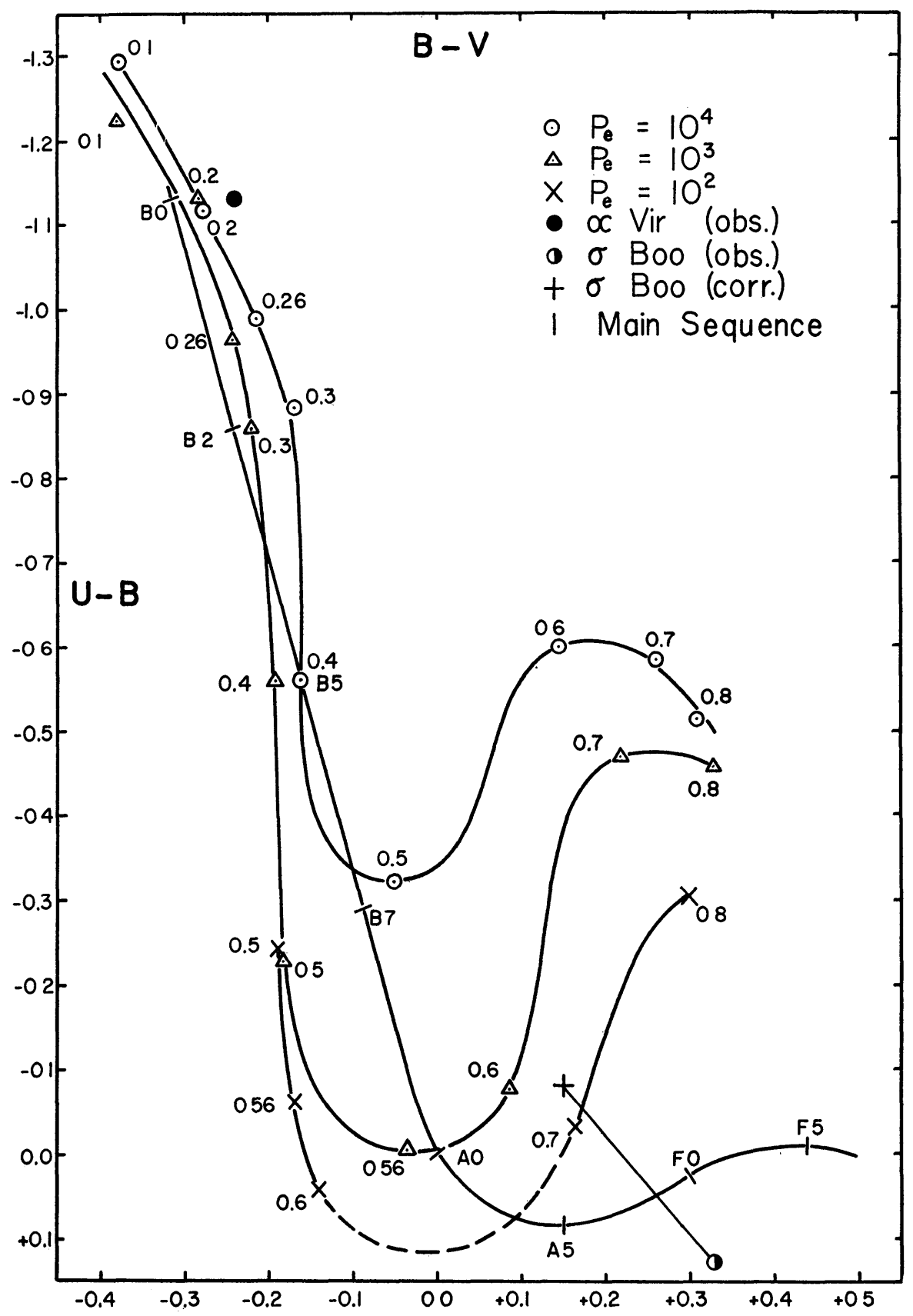

Fig. 1. - The observed $U-B$ and $B-V$ colors are on the curve labeled with main-sequence spectral types. The theoretical colors for three electron pressures, $P_{e}$, are given. The zero point is set by $\alpha$ Vir and $\sigma$ Boo; the latter is shown both with and without line corrections The theoretical curves are labeled according to $\theta_{e}$. 
Of course, this relation is similar to that previous $\theta_{e}, \theta_{c}$ relation, except that we have now included the effect of the Balmer lines. The results for $B-V$ colors at three electron pressures are plotted in Figure 4; they show that the $B-V$ colors give a base line such that the deviation between color and effective temperatures is large and pressure-sensitive. Over almost the entire range, the $B-V$ colors indicate apparently higher color temperature than the star's effective temperature would predict. (At about $\theta \gtrsim 0.6$ the metallic lines, not included in our computation, would appear and reduce the color tem-

TABLE 2

COMPUTED FLUXes AND $B-V$ AND $U-B$ COLORS

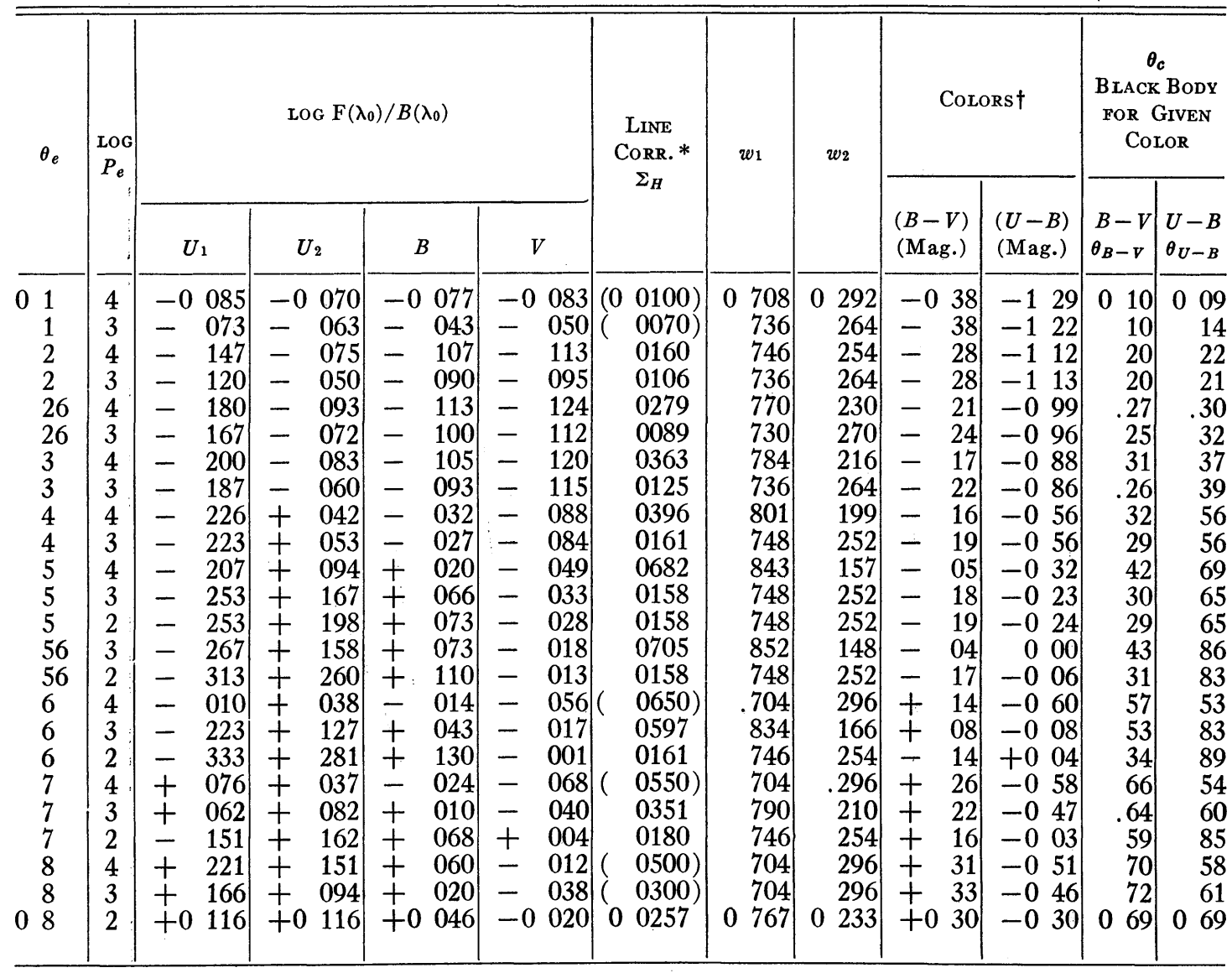

* Numbers in parentheses are extremely rough estimates

$\dagger$ The $B-V$ and $U-B$ colors are here computed on the zero points $c_{2}^{\prime}=+0.520$ and $c_{3}^{\prime}=-0335$.

perature, i.e., bring the star closer to the black-body line.) The one curve plotted to give the color temperature based on $U-B$ colors, $\theta_{U-B}$, shows the opposite deviation, caused by the Balmer jump. It is apparent that the three-color behavior of a star gives very important clues to its physical nature for stars in which hydrogen is the dominant source of opacity. More refined analysis, such as that by Osawa (1956), gives greater certainty to some of the conclusions of this investigation. For stars with abnormal surface gravity (e.g., population II objects) or with an abnormal abundance ratio of hydrogen/metals (e.g., globular-cluster stars and the subdwarfs), deviations from the normal $U-B$ and $B-V$ relation are to be expected and give quantitative results of interest (Sandage 1956). 


\section{COLORS OF WHITE DWARFS}

One very direct application of the technique developed concerns the effects of lines on the colors of white dwarfs. A few white dwarfs have been observed (Johnson and Morgan 1953, Fig. 9), and unpublished data for nearly all white dwarfs have been supplied by D. L. Harris III. The white dwarfs are found to cluster around and above the straight line for a black body drawn in Figure 3; the bluest are near $B-V=-0.15$ and the majority near $B-V=0.00$; the Balmer jump has apparently disappeared, since the

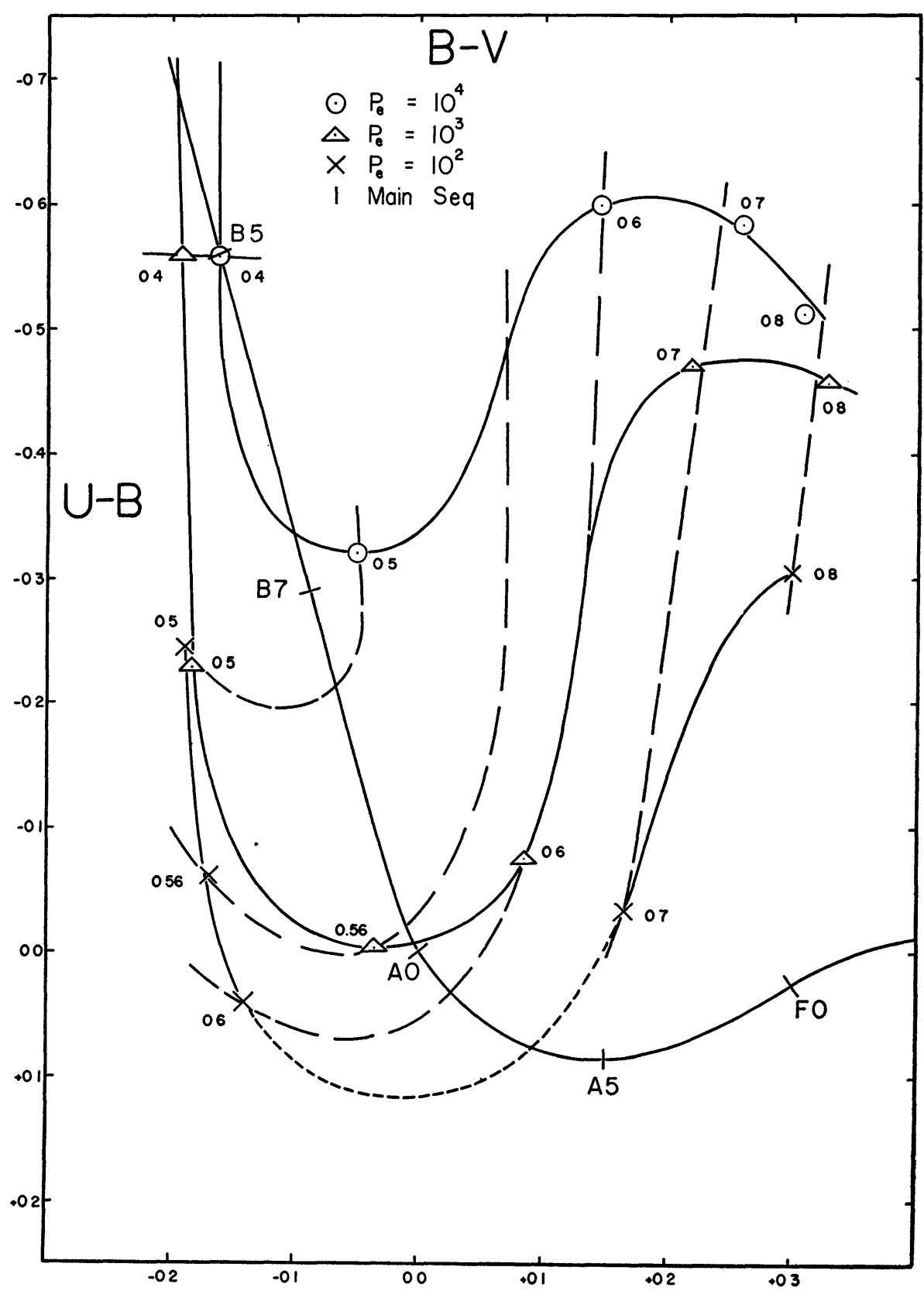

Fig. 2.-An enlarged portion of Fig. 1, in the range of spectral type B5-F0, is shown. The long dashed curves show loci of constant $\theta_{e}$; the short dashed curve represents the uncertainty in the location of the region of maximum Balmer discontinuity. 
$U-B$ colors range from about 0.4 to $0.8 \mathrm{mag}$., abnormally negative for the observed $B-V$ color. Inspection of Figures 1 and 2 indicates that the disappearance of the Balmer jump would require extremely high electron pressures; Figures 3 and 4 would tend to show that the hottest white dwarf would have $\theta_{e} \approx \theta_{B-V}$ and temperatures less than $15000^{\circ} \mathrm{K}$. Both these conclusions seem incorrect.

However, measurement of the equivalent widths of the hydrogen lines in a typical white dwarf (Greenstein 1956) shows that the Balmer jump exists but that it sets in at

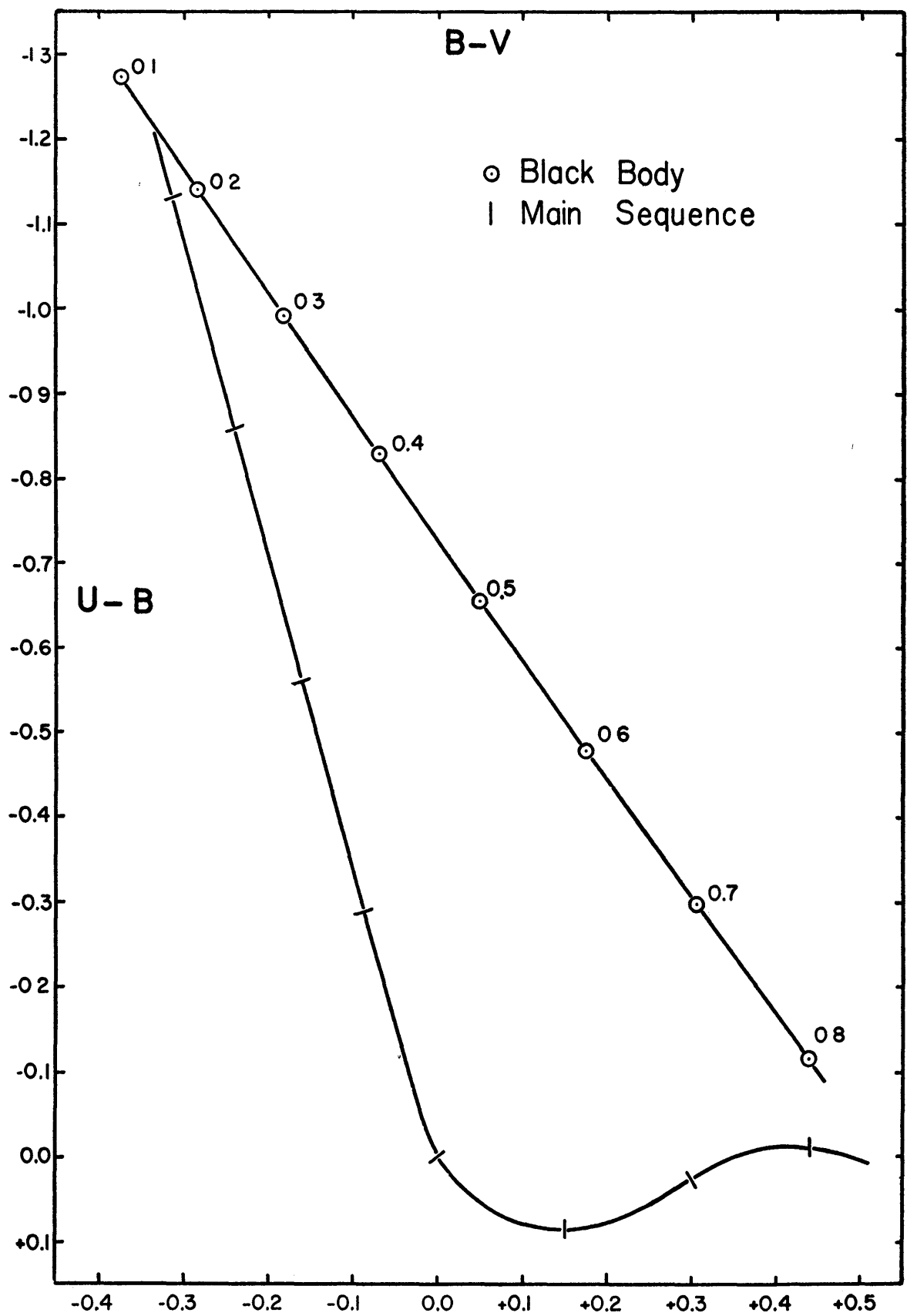

FIG. 3. - The observed colors along the main sequence are compared with the predicted colors of a black body; the latter are labeled according to $\theta_{e}$. Spectral types on lower curve same as in Fig. 1 . 
about $3850 \mathrm{~A}$ and that the hydrogen lines are extremely strong, up to $30 \mathrm{~A}$ equivalent width for $H \gamma$. Thus the $U$ band should be treated as lying completely within the Balmer continuum, the $B$ band should have a very substantial correction for lines, and the $V$ band is hardly affected. Using the measured strengths of the Balmer lines, the line corrections in equations (13) and (14) are found empirically to be as shown in Table 3.

The photoelectric colors quoted are preliminary, largely due to D. L. Harris III and kindly communicated in advance of publication. These results are plotted in Figure 5, which shows that a purely colorimetric approach is insufficient for the white dwarfs.

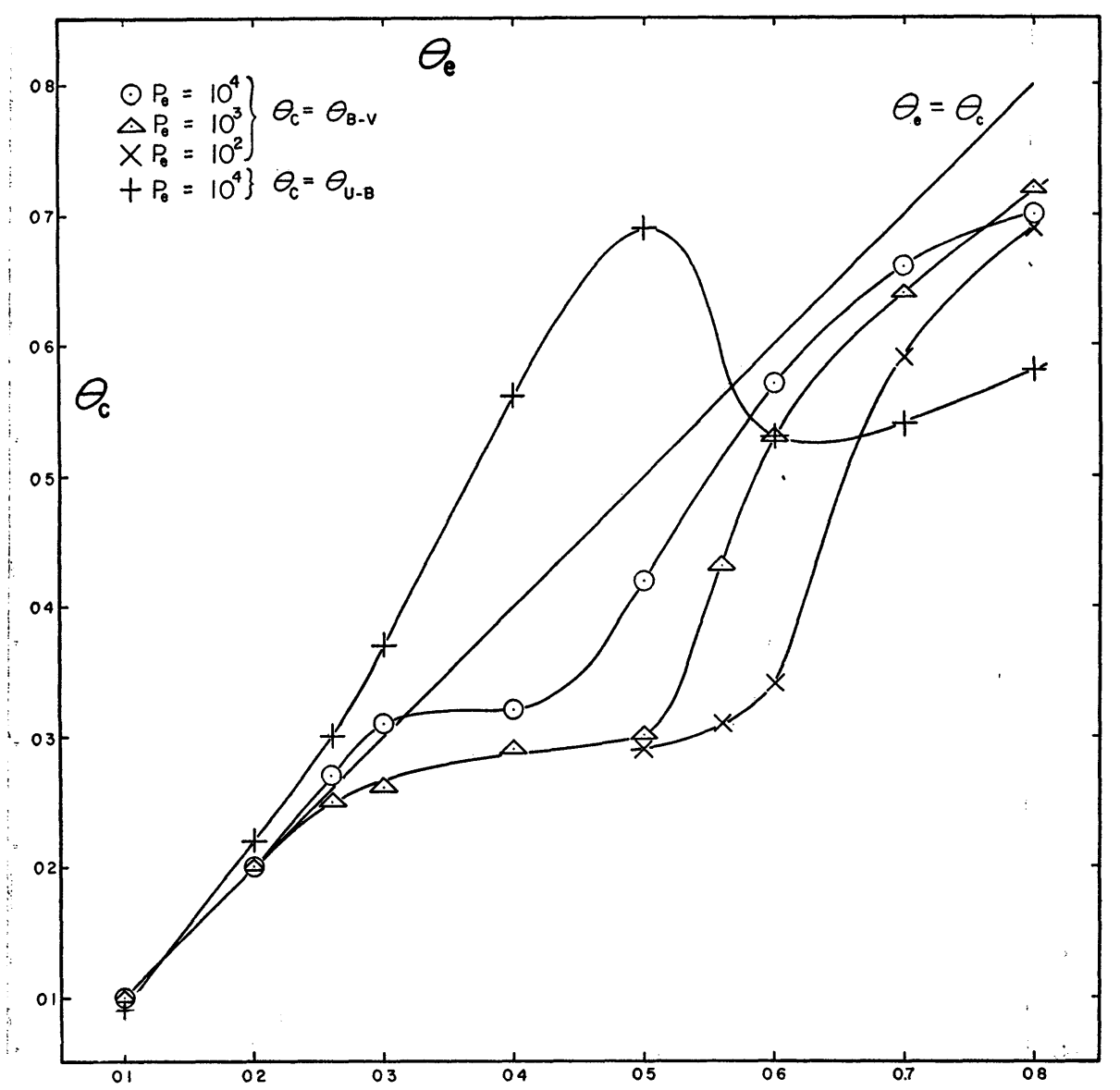

FIG. 4:-The relations between effective and color temperatures. The three curves which lie below the $45^{\circ}$ slope $\left(\theta_{e}=\theta_{e}\right)$ give the color temperature $\theta_{B^{-V}}$ of a black body which has the same $B-V$ color as that predicted for a star of $\theta_{e}$. The upper curve, labeled with crosses, gives the color temperature $\theta_{U-B}$ when the match is made with the $U-B$ color.

The vectors representing the effect of absorption lines move about half of the white dwarfs from near the black-body line to the left of the locus of $B-V$ and $U-B$ for the main sequence, while another half, those having weak or no lines, are hardly affected at all, merely approaching the black-body-curve more closely. The apparent temperatures to be derived from $B-V$ are greatly increased for some white dwarfs-approaching $25000^{\circ} \mathrm{K}$-while no change is produced in the temperature scale for white dwarfs with weak hydrogen lines. The fact that some white dwarfs, when corrected, are below and to the left of the main sequence does not mean that the surface gravity is low. The process of removal of the flux absorbed by lines must be balanced physically by a re- 


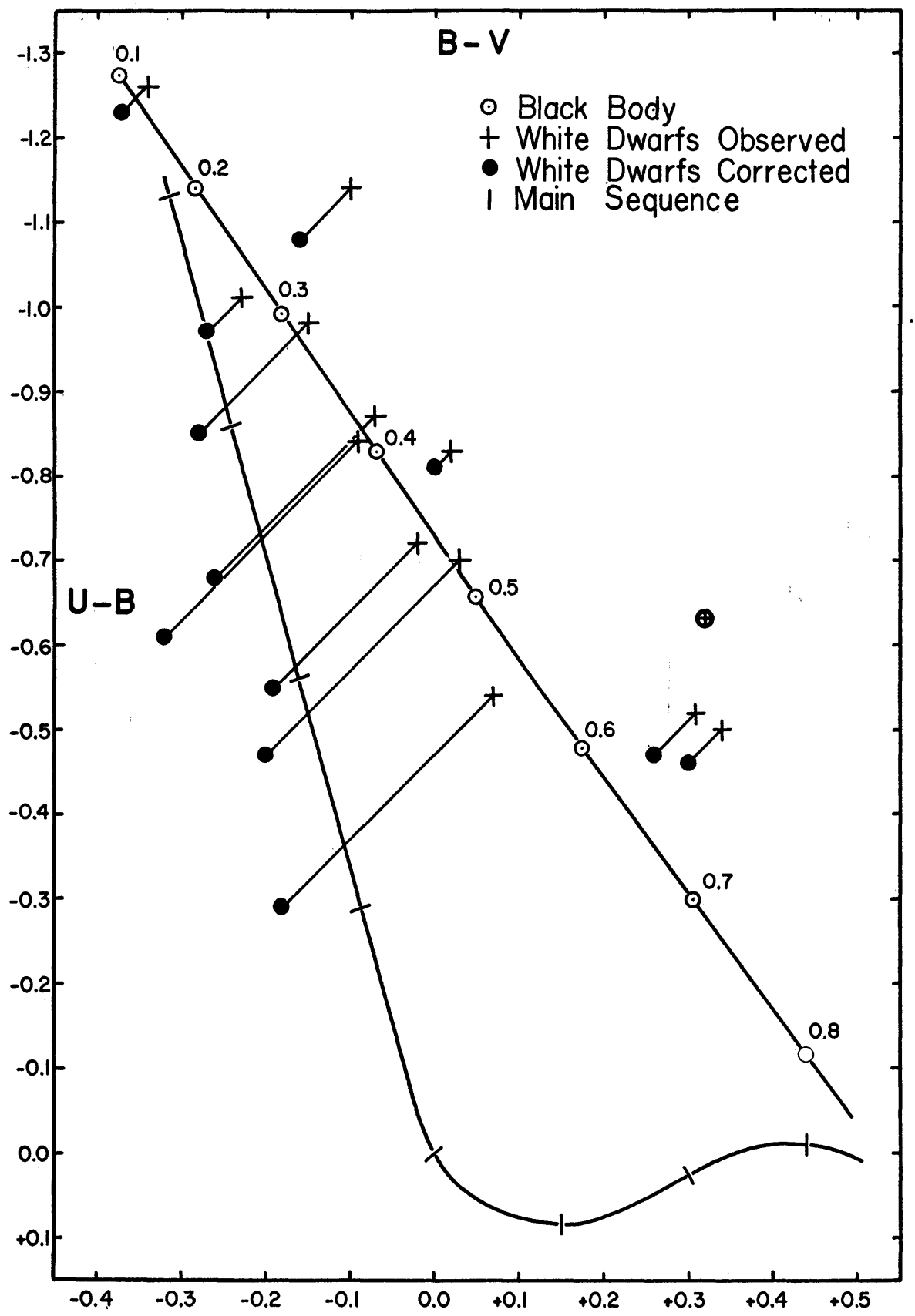

Fig. 5.-The effect of the Balmer lines on the colors of white dwarfs. The main-sequence and the black-body curves are repeated from Fig. 3. The crosses are the observed colors of white dwarfs, the filled circles the position after correction of the $B$ magnitude for the lines Note that the weak-line white dwarfs lie close to the black body. 
emission elsewhere, caused by the effect of the blanketed radiation, and the correction made gives only a first-order indication of the true color-temperature of the real star.

The corrections here discussed are important for the theory of white dwarfs but are even more significant in demonstrating the need for a combined spectroscopic and colorimetric study. Similar effects but of opposite sign are known to occur in the F-type subdwarfs.

TABLE 3

EFFECT OF LINES ON THE COLORS OF WHITE DWARFS

\begin{tabular}{|c|c|c|c|c|}
\hline \multirow{2}{*}{ Star } & \multicolumn{2}{|c|}{ ObSeRved } & \multicolumn{2}{|c|}{ Corrected } \\
\hline & $\begin{array}{c}B-V \\
\text { (Mag.) }\end{array}$ & $\begin{array}{c}U-B \\
\text { (Mag.) }\end{array}$ & $\begin{array}{c}B-V \\
\text { (Mag.) }\end{array}$ & $\begin{array}{c}U-B \\
\text { (Mag.) }\end{array}$ \\
\hline $\begin{array}{l}\text { BD+28 } 4211 \\
\text { HZ } 29 \\
\text { L } 1244-26 \\
\text { HZ } 43 \\
\text { Grw }+70^{\circ} 5824 \\
\text { Wolf } 1346 \\
\text { Grw }+82^{\circ} 3818 \\
\text { LDS:678A } \\
40 \text { Eri B.. } \\
\text { SA 29-130 } \\
\text { Ross } 627 \\
\text { L 870-2. } \\
\text { L } 745-46 .\end{array}$ & $\begin{array}{l}-034 \\
-\quad 23 \\
-\quad 15 \\
-\quad 10 \\
-\quad 09 \\
-\quad 07 \\
-\quad 02 \\
+\quad 02 \\
+\quad 03 \\
+\quad 07 \\
+\quad 31 \\
+\quad 34 \\
+0\end{array}$ & $\begin{array}{ll}-1 & 26 \\
-1 & 01 \\
-0 & 98 \\
-1 & 14 \\
-0 & 84 \\
-0 & 87 \\
-0 & 72 \\
-0 & 83 \\
-0 & 70 \\
-0 & 54 \\
-0 & 52 \\
-0 & 50 \\
-0 & 63\end{array}$ & $\begin{array}{ll}-0 & 37 \\
- & 27 \\
- & 28 \\
- & 16 \\
- & 32 \\
- & 26 \\
- & 19 \\
& 00 \\
- & 20 \\
- & 18 \\
+ & 26 \\
+ & 30 \\
+0 & 32\end{array}$ & $\begin{array}{ll}-1 & 23 \\
-0 & 97 \\
-0 & 85 \\
-1 & 08 \\
-0 & 61 \\
-0 & 68 \\
-0 & 55 \\
-0 & 81 \\
-0 & 47 \\
-0 & 29 \\
-0 & 47 \\
-0 & 46 \\
-0 & 63\end{array}$ \\
\hline
\end{tabular}

\section{REFERENCES}

Barbier, D., and Chalonge, D. 1939, Ann d'ap., 2, 254.

Barbier, D., and Divan, L. 1952, Ann. d'ap, 15, 201.

Burkhardt, G. 1936, Zs. f. A p., 13, 56.

Chandrasekhar, S. 1950, Radiative Transfer (Oxford: Clarendon Press), p 307.

Chandrasekhar, S., and Münch, G. 1946, A p. J., 104, 446.

Code, A D. 1954, N.S.F. Conference on Stellar Atmospheres, ed. Wrubel (Indiana University), pp. 14-19. 1955, private communication.

Greenstein, J. L. 1956, Third Berkeley Symposium on Mathematical Statistics and Probability, ed. Neyman (Berkeley) , 3, 11-29; also unpublished data.

Günther, S. 1933, Zs.f. Ap., 7, 106.

Johnson, H. L., and Morgan, W. W. 1951, Ap. J., 114, 522.

-. 1953, ibid., 117, 313.

Keenan, P. C., and Morgan, W. W. 1951, Astrophysics, ed Hynek (New York: McGraw-Hill Book Co., Inc.), pp. $20 \mathrm{ff}$.

Münch, G. 1945, $A$ p. $J, 102,385$.

Osawa, K. 1956, Ap.J., 123, 513.

Sandage, A. 1956; $A$ p. J., $123,278$.

Schwarzschild, M., Searle, L., and Howard, R. 1955, Ap. J , 122, 353.

Stock, J. 1956, Ap. J., 123, 253.

Ueno, S. 1954, Contr. Inst. Ap, U. Kyoto, No. 42.

Ueno, S., Saito, K., and Jugaku, J 1954, Contr. Inst. Ap, U. Kyoto, No. 43.

Vitense, E. 1951, $Z s . f . A p, 28,81$. The abundances used were, by number of atoms, metals $=1$, hydrogen $=4500$; among the lighter elements, $\mathrm{He}=800, \mathrm{C}+\mathrm{N}+\mathrm{O}=8, \mathrm{Ne}=5$. 